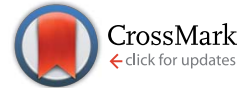

Cite this: J. Mater. Chem. A, 2016, 4 , 6368

Received 29th January 2016 Accepted 29th March 2016

DOI: $10.1039 / c 6 t a 00900 j$

www.rsc.org/MaterialsA

\section{UV-absorbing cellulose nanocrystals as functional reinforcing fillers in polymer nanocomposite films}

\begin{abstract}
Juho Antti Sirviö, ${ }^{\star a}$ Miikka Visanko, ${ }^{a}$ Juha P. Heiskanen $^{\mathrm{b}}$ and Henrikki Liimatainen ${ }^{a}$
Reinforcing, surface-functionalized cellulose nanocrystals (CNCs) with photoactive groups were obtained from wood cellulose fibers using sequential periodate oxidation and a "click-type" reaction between aldehyde groups and $p$-aminobenzoic acid in an aqueous environment, followed by mechanical disintegration. In the solution state, CNCs exhibited very high UV-absorption properties, especially in UVA and UVB regions (100\% absorption was achieved with only $0.1 \%$ of CNCs) and high transparency in the visible light region (around $90 \%$ with $0.1 \%$ of CNCs). The fabricated CNCs functioned as lightweightreinforcing fillers with high UV-absorption capability when incorporated into a poly(vinyl alcohol) (PVA) matrix. Complete UVA and UVB opacity of the nanocomposite was achieved using $10 \%$ of CNCs while simultaneously retaining over $80 \%$ transparency over the whole visible light spectrum. In addition, up to $33 \%$ and $77 \%$ higher tensile strength and modulus, respectively, were achieved using $10 \%$ of CNCs compared to pristine PVA. This result presented a unique way to produce multifunctional CNCs to be incorporated into nanocomposite structures instead of metal nanoparticles. These CNCs are supposed to be suitable for many applications requiring high visible light transparency and blocking of UV radiation.
\end{abstract}

\section{Introduction}

The addition of functional fillers can significantly improve the properties of organic transparent polymers, as they confer new features on the materials, including magnetoelasticity, ${ }^{1}$ a high reflectance index, and absorption of specific wavelengths of light (e.g., UV). ${ }^{2}$ UV-absorption properties are important in many applications, as although the energy of UV light is too low to cause ionization, it can accelerate many chemical reactions, causing severe damage to animals, plants, ${ }^{3}$ and materials. ${ }^{4}$ In particular longer wavelength UVB $(280-315 \mathrm{~nm})$ and UVA (315$400 \mathrm{~nm}$ ) are important from a biological perspective, as they are not or only partially adsorbed by the ozone layer, whereas shorter wavelength UVC $(100-280 \mathrm{~nm})$ is completely eliminated at the stratosphere. ${ }^{5}$ To prevent UV light induced damage, highly transparent UV-absorption materials are required, for example, in health science, such as in ophthalmology. ${ }^{6}$ The high transparency of a polymer matrix can be retained by using nanoscale-sized fillers, which do not absorb and scatter visible light. Most functional fillers are composed of inorganic nanoparticles, such as $\mathrm{ZnO}$ or $\mathrm{TiO}_{2} .{ }^{1}$ Although inorganic nanoparticles that block UV light have several advantages, including a long life cycle, there are health and environmental concerns surrounding their use. For example, the leaching of inorganic

${ }^{a}$ Fiber and Particle Engineering, University of Oulu, P.O. Box 4300, FI-90014, Finland. E-mail: juho.sirvio@oulu.fi

${ }^{b}$ Research Unit of Sustainable Chemistry, University of Oulu, P.O. Box 3000, FI-90014 Oulu, Finland nanoparticles is a potential health hazard due to their toxicity and possible bioaccumulation. ${ }^{7-9}$ Inorganic nanoparticles may also induce photodegradation of polymers. ${ }^{10}$ In addition, some inorganic nanoparticles are produced using costly metals, which decrease their economic and ecological viability.

Nanocellulose, which can be isolated from numerous cellulosic sources, is a relatively novel class of biomaterials. ${ }^{11}$ Long cellulose nanofibers (CNFs) are mostly obtained by mechanical disintegration of natural or chemically modified cellulose fibers. ${ }^{12}$ Short and rod-like cellulose nanocrystals (CNCs) are produced by acid hydrolysis of amorphous parts of cellulose. ${ }^{13}$ Several acid-free methods of CNC production also exist. ${ }^{14-17}$ Both CNFs and CNCs are promising materials for many highend applications, as they are lightweight, biodegradable, have a large surface area, and are produced from cellulose, which is a renewable and abundant biopolymer. ${ }^{18}$ They are also relatively nontoxic, although their toxicity depends on the production method (i.e., the chemistry applied during the preparation). ${ }^{19}$

Due to the lightweight and high strength of CNFs and CNCs, they have been studied as organic filler materials for synthetic and natural nanocomposites. ${ }^{20}$ So far most of the research has concentrated on improving the mechanical properties of nanocomposites by exploiting the high reinforcing effect of nanocellulose. However, the potential of nanocellulose as a bifunctional UV-absorbing and reinforcing filler in composites has not been widely reported. UV-absorbing nanocomposites containing CNCs with lignin ${ }^{21}$ and CNFs with $\mathrm{ZnO}$ nanoparticles ${ }^{22}$ have been produced, but their UV absorption was based on the presence of lignin or ZnO nanoparticles. 
Postchemical polymerization of CNCs with polyrhodanine was shown to alter visible light absorption properties of CNCs depending on the $\mathrm{pH}$ and polyrhodanine-functionalized CNCs were proposed as $\mathrm{pH}$ indicators. ${ }^{23}$ A CNF aerogel with photoswitchable water absorption properties was studied, but the photoactive properties of the gel were due to a $\mathrm{TiO}_{2}$ coating. ${ }^{24}$

Herein, we report the fabrication of photoactive CNCs based on a "click-type" reaction between $p$-aminobenzoic acid (PABA) and periodate-oxidized cellulose. CNCs were then used in nanocomposite films together with poly(vinyl alcohol) (PVA) as the polymer matrix. The aim of the study was to investigate the properties of CNCs produced by this method and their use as a UV absorbing reinforcement agent for PVA. In particular, the effect of the amount of CNCs (0-10\%) on the properties of PVA was investigated.

\section{Experimental}

\section{Materials}

Bleached birch pulp (Betula pendula) was obtained as dry sheets and used as a cellulose material after disintegration in deionized water. The p.a. grade chemicals used for the periodate oxidation $\left(\mathrm{NaIO}_{4}\right)$, the aldehyde content analyses $\left(\mathrm{NH}_{2} \mathrm{OH} \cdot \mathrm{HCl}\right)$ and the fabrication of nanocomposite films (PVA (Mowiol 3-96, 96.8-97.6 mol\% hydrolysis)) were obtained from Sigma-Aldrich (Germany). p-Aminobenzoic acid was obtained from TCI (Germany). All the chemicals were used as received, without further purification. Deionized water was used throughout the experiments.

\section{Synthesis and fabrication of $\boldsymbol{p}$-aminobenzoic acid modified cellulose nanocrystals}

The pulp sample was oxidized with sodium metaperiodate by weighing $12.0 \mathrm{~g}$ (dry weight) of cellulose in a $2000 \mathrm{ml}$ flask and adding $1200 \mathrm{ml}$ of deionized water and $9.84 \mathrm{~g}$ of $\mathrm{NaIO}_{4}$. The reaction vessel was covered with aluminum foil to prevent photoinduced decomposition of periodate, and the mixture was stirred with a magnetic stirrer in a water bath at $75{ }^{\circ} \mathrm{C}$, together with $21.6 \mathrm{~g}$ of $\mathrm{LiCl}$ as an additive. After $3 \mathrm{~h}$, the product was filtered and washed several times with deionized water to remove iodine-containing compounds. Undried periodateoxidized pulp was then allowed to react with sodium 4-aminobenzoate (10 times molar excess compared to the aldehyde groups) for $72 \mathrm{~h}$ at $\mathrm{pH} 5.8 \mathrm{using}$ dilute $\mathrm{HCl}$ solution. After the reaction, the product was filtered and washed with deionized water. The washed pulp was diluted to $2 \%$ with water, and the $\mathrm{pH}$ was adjusted to 7 by adding a few drops of dilute $\mathrm{NaOH}$ solution, followed by further dilution to $1 \%$ using water. The sample was then disintegrated using a microfluidizer (Microfluidics M-110EH-30), with one pass through 400 and $200 \mu \mathrm{m}$ chambers at a pressure of 1300 bar, then passed once through 400 and $100 \mu \mathrm{m}$ chambers at a pressure of 2000 bar, and finally passed once through 200 and $87 \mu \mathrm{m}$ chambers at a pressure of 2000 bar. Reference CNCs with carboxylic acids were prepared according to the reported methodologies based on our previous study. ${ }^{25}$

\section{Optical transmittance of cellulose nanocrystal solutions}

Diluted solutions (0.1-0.5\%) were prepared using water and UVVis spectra (190-800 nm) were recorded with a UV-visible spectrometer (Shimadzu, Japan) using quartz cuvettes.

\section{${ }^{1} \mathrm{H}$ NMR characterization of $\boldsymbol{p}$-aminobenzoic acid modified cellulose nanocrystals}

A sample of PABA modified CNCs $(10 \mathrm{mg})$ was dissolved in $d_{6}$ DMSO and placed in a $5 \mathrm{~mm}$ NMR tube. The ${ }^{1} \mathrm{H}$ NMR spectrum was recorded by using a Bruker Ascend $400 \mathrm{MHz}$ spectrometer at ambient temperature.

\section{Transmission electron microscopy}

The morphology of the fabricated CNCs was analyzed using a Tecnai G2 Spirit (FEI Europe, Eindhoven, The Netherlands) transmission electron microscope. Samples were prepared by diluting each sample with Milli-Q water. A small droplet of the dilution was placed on top of a Butvar-coated copper grid. Any excess amount of the sample was removed from the grid by touching the droplet with a corner of filter paper. The samples were negatively stained by placing a droplet of uranyl acetate $(2 \% \mathrm{w} / \mathrm{v})$ on top of each specimen. Excess uranyl acetate was removed with filter paper, as described above. The grids were dried at room temperature and analyzed at $100 \mathrm{kV}$ under standard conditions. Images were captured using a Quemesa CCD camera, and the width and length of the CNCs were measured using iTEM (Olympus Soft Imaging Solutions GMBH, Munster, Germany) image analysis software. In total, 50 CNCs of each sample were measured. The final results were averaged, and standard deviations were calculated.

\section{X-ray diffraction}

The crystalline structure of the PABA modified CNCs was investigated using wide-angle X-ray diffraction (WAXD). Measurements were conducted on a Rigaku SmartLab 9 kW rotating anode diffractometer (Japan) using a Co $\mathrm{K} \alpha$ radiation $(40 \mathrm{kV}, 135 \mathrm{~mA})(\lambda=1.79030 \mathrm{~nm})$. Samples were prepared by pressing tablets of freeze-dried celluloses to a thickness of 1 $\mathrm{mm}$. Scans were taken over a $2 \theta$ (Bragg angle) range from $5^{\circ}$ to $50^{\circ}$ at a scanning speed of $10^{\circ} \mathrm{s}^{-1}$, using a step of $0.5^{\circ}$. The degree of crystallinity in terms of the crystallinity index (CrI) was calculated from the peak intensity of the main crystalline plane (200) diffraction $\left(I_{200}\right)$ at $26.2^{\circ}$ and from the peak intensity at $22.0^{\circ}$ associated with the amorphous fraction of cellulose $\left(I_{\mathrm{am}}\right)$, according to eqn (1): ${ }^{26}$

$$
\mathrm{CrI}=\left(\frac{I_{200}-I_{\mathrm{am}}}{I_{200}}\right) \times 100 \%
$$

It should be noted that due to the $\mathrm{Co} \mathrm{K} \alpha$ radiation source, the cellulose peaks have different diffraction angles compared to results obtained with the $\mathrm{Cu} \mathrm{K} \alpha$ radiation source. 


\section{Fabrication of the nanocomposite films}

First, $0.88 \mathrm{~g}$ of PVA was dissolved in deionized water at $90{ }^{\circ} \mathrm{C}$ in a round-bottomed flask. After allowing the solution to cool, the cellulose nanocrystals were added (0.1-10\%, depending on the amount of PVA). The solution was degassed under vacuum suction, followed by casting on a polystyrene tray. The films were allowed to dry at room temperature for five days.

\section{Optical transmittance of the nanocomposite films}

The transmittance (190-800 $\mathrm{nm}$ ) of the nanocomposites was measured with a UV-Vis-NIR spectrophotometer (Carry 500 Scan, Varian Inc., USA), with a step size of $1 \mathrm{~nm}$.

\section{Mechanical properties of the nanocomposites}

Tensile tests were performed with a universal material testing machine (Instron 5544, USA), equipped with a $100 \mathrm{~N}$ load cell. The PVA-RCNC films were cut into thin strips, with each specimen having a width of $5 \mathrm{~mm}$. For the tensile testing, a $40 \mathrm{~mm}$ gauge length was used at a strain rate of $4 \mathrm{~mm} \mathrm{~min}^{-1}$, and six specimens were tested. Using a Hanatek Precision Thickness Gauge Model FT3 (Rhopoint Instruments, UK) thickness tester, the thickness of each specimen was determined as the average of three random locations on the specimen. The film thicknesses ranged from 14-44 $\mu \mathrm{m}$. The tests were conducted under $50 \%$ relative humidity at a temperature of $23{ }^{\circ} \mathrm{C}$ and under a prestrain of $0.05-0.1 \mathrm{~N}$. The specimens were conditioned for one day in the measurement environment before testing. Young's modulus was calculated from the initial linear portion of the stress-strain curve, and the ultimate tensile strength was defined as the stress under which the specimen broke.

\section{Results and discussions}

In this study, pre-chemical modification of wood cellulose followed by mechanical disintegration was used to fabricate CNCs with UV absorption properties. First, the cellulose was oxidized to dialdehyde cellulose (DAC) with an aldehyde content of 3.85 mmol $\mathrm{g}^{-1}$ (ref. 27) prior to the cellulose modification with an amine. In the second step, DAC was then reacted with a sodium salt of PABA in a slightly acidic ( $\mathrm{pH}$ 5.8) aqueous environment to attach the photoactive groups to the cellulose. PABA was chosen because it exhibits low toxicity and an absorption maximum of 290-320 $\mathrm{nm}$ in the UVB region (absorption is due to the aromatic benzene group with amine and carboxylic acid functionalities ${ }^{28}$ ). The amino group of PABA is attached to an aromatic ring, which allows the formation of a conjugated imine group by the reaction with aldehyde. Conjugated imine groups are more stable in an aqueous environment than their nonconjugated counterparts, with conjugation also potentially enhancing the light absorption properties. ${ }^{29}$ Consequently, no reducing agent was required to attach PABA to DAC. The formation of PABA-cellulose (PABAC) can be considered as a "click-type" reaction, as demonstrated in the synthesis route shown in Fig. 1a. The reaction needs only an acid catalyst (water is produced as a by-product), thus increasing the atom economy of the synthesis of photoactive CNCs.
According to the elemental analysis of nitrogen, the PABA group content of PABAC was $0.43 \mathrm{mmol} \mathrm{g}^{-1}$, indicating that around $12 \%$ of the aldehyde groups reacted with PABA. The aldehyde-modification degree was similar to previously reported values for alkylamine-modified ${ }^{30}$ and bisphosphonatemodified $\mathrm{CNCs}^{31}$ obtained by reductive amination of DAC. However, unlike CNCs obtained by reductive amination, the residual aldehydes are not converted to hydroxyl groups but instead appear as free aldehydes or bound to water or hydroxyl groups of cellulose in hydrate or hemiacetal functionality, respectively. ${ }^{32}$ The residual aldehydes can be utilized in further surface modification (attachment of other chemical groups, such as cross-linking with diamine, ${ }^{33}$ or as reducing groups in nanoparticle synthesis ${ }^{34}$ ). In addition, aldehydes might also serve as reactive groups for cross-linking with the polymer matrix (amine $e^{35}$ or hydroxyl groups ${ }^{25}$ containing polymers, see the results of the tensile strength measurements below). It should be noted that the residual aldehydes may decrease the stability of cellulosic materials as aldehydes containing cellulose is alkaline labile; ${ }^{36}$ however, no significant effect on the thermal stability has been observed for DAC. ${ }^{37}$

Chemical modification of cellulose with PABA was studied using diffusion reflectance infrared Fourier transform spectroscopy (DRIFT). After periodate oxidation, a typical $\mathrm{C}=\mathrm{O}$ stretching vibration band of aldehyde was observed at a wavenumber of $1730 \mathrm{~cm}^{-1}$ (Fig. 1b). ${ }^{32}$ This band disappeared after modification with PABA, indicating that the aldehydes reacted with amines to form imines. As shown by an elemental analysis, the aldehydes were only partly modified by PABA, and the remaining aldehydes were masked by the formation of hemiacetals or hydrates after the reaction with hydroxyl groups of cellulose or water, respectively. ${ }^{32}$ Aromatic in-ring C-C stretching vibration was seen at $1606 \mathrm{~cm}^{-1}$, whereas asymmetric vibration of deprotonated carboxylic acid was observed at a wavenumber of $1547 \mathrm{~cm}^{-1}$. Characteristic aromatic imine bond vibration was observed as a shoulder around $1640 \mathrm{~cm}^{-1} \cdot{ }^{38}$ This is also the wavenumber of the typical water band observed in cellulose-based materials, which most likely caused the poor separation of the imine band. The shoulder might also be superimposed on the remaining aldehyde and imine bands.

PABA modified CNCs were also studied using ${ }^{1} \mathrm{H}$ NMR spectroscopy. The local zoom of the ${ }^{1} \mathrm{H}$ NMR spectrum (4.00$9.00 \mathrm{ppm})$ is presented in Fig. 1c. Typical obscure cellulose proton signals are observed at $\delta_{\mathrm{H}} 4.31,4.70$ and $5.44 \mathrm{ppm} .{ }^{16}$ The aryl proton signals of the PABA substituent can be observed as two clear doublets at $\delta_{\mathrm{H}} 6.50$ and $7.58 \mathrm{ppm}$. Moreover, the sharp singlet at $\delta_{\mathrm{H}} 8.54 \mathrm{ppm}$ is assigned to the imine proton ${ }^{39-41}$ which confirms the presence of a covalent bond between the PABA substituent and cellulose backbone.

After chemical modifications, a 1\% PABAC suspension was prepared, and the $\mathrm{pH}$ was adjusted to 7 prior to liberation of the CNCs through mechanical disintegration of the modified cellulose. Due to the presence of ionized carboxylic acid groups, the PABAC disintegrated easily to CNCs. The ionized carboxylic acid groups created repulsion between the cellulose fibers, improving the disintegration efficiency. The TEM images presented in Fig. 2 confirm the formation of CNCs after the 


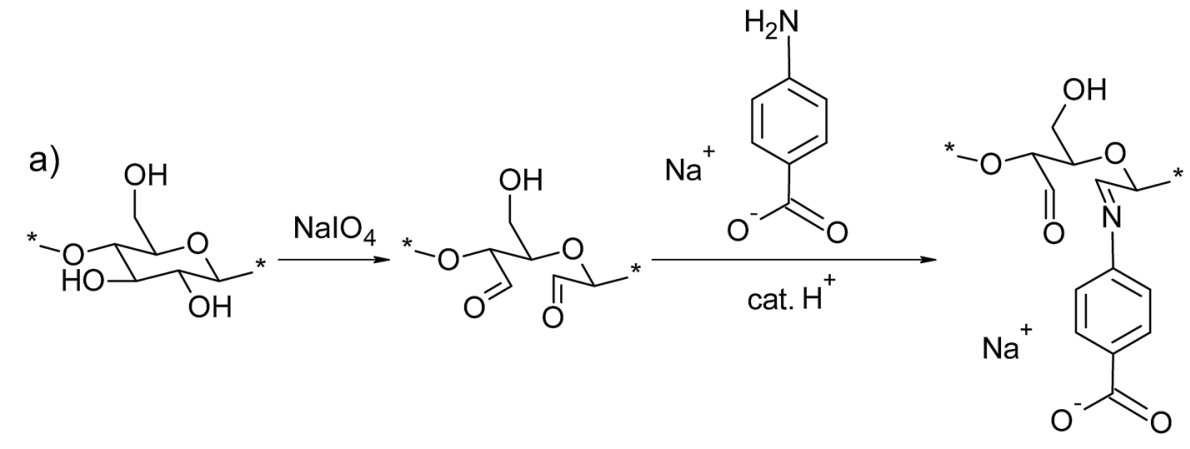

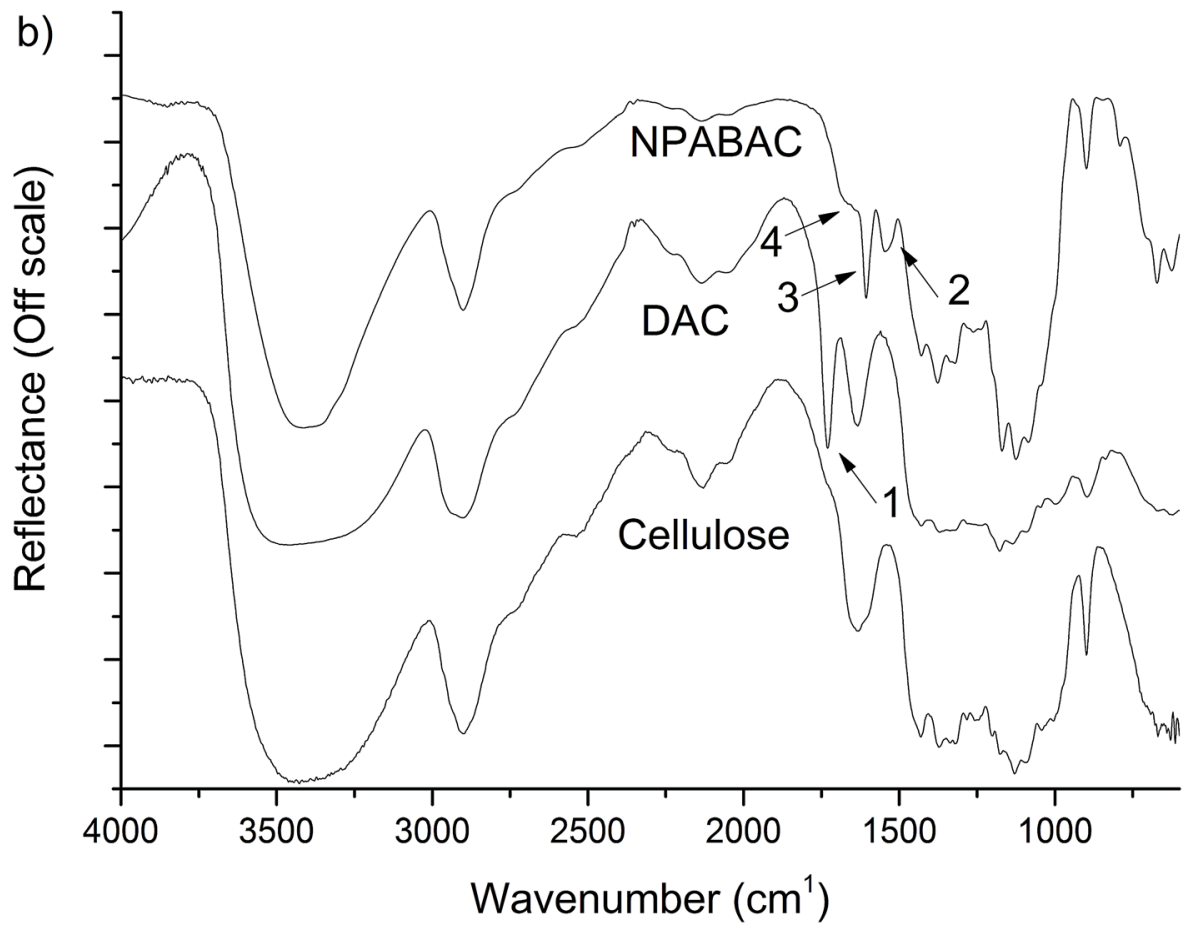

C)

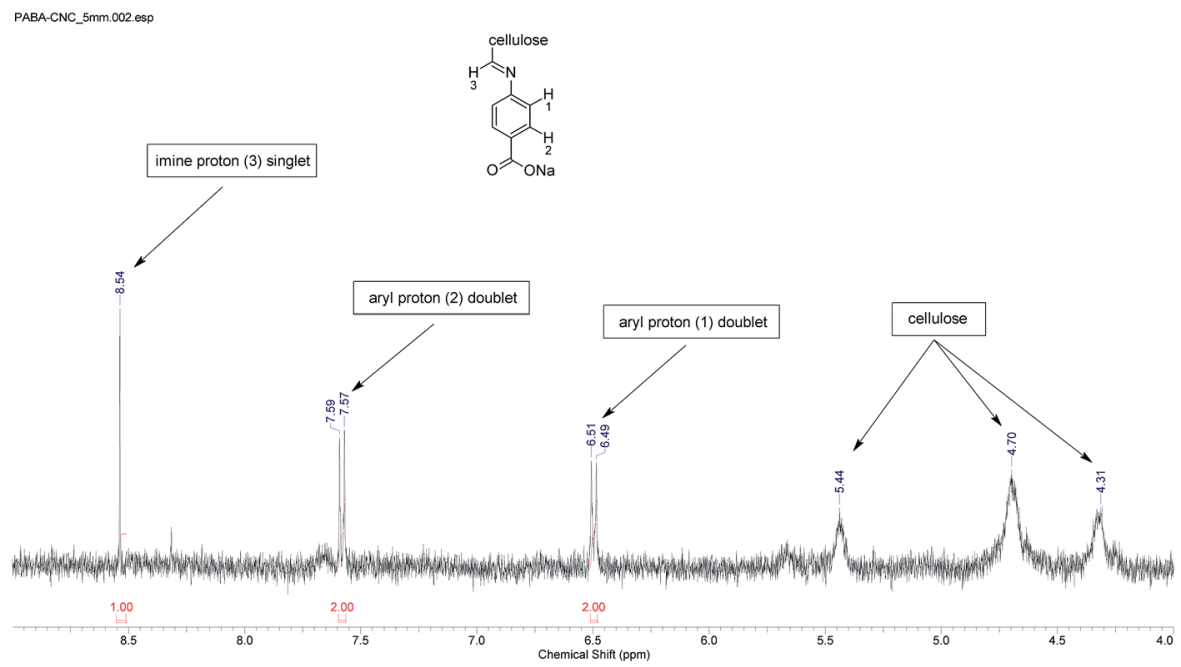

Fig. 1 (a) Schematic illustration of the synthesis of para-aminobenzoic acid-modified cellulose (PABAC) using dialdehyde cellulose (DAC) and a "click-type" reaction (for the sake of clarity, the second aldehyde is drawn in a free form; however, most likely, it forms bonds with the water or hydroxyl groups of cellulose in the hydrates or hemiacetals, respectively). (b) DRIFT spectra of cellulose, DAC, and PABAC. The most distinguishable bands of the chemically modified cellulose are numbered: (1) $\mathrm{C}=\mathrm{O}$ of aldehyde, (2) COO- of deprotonated carboxylic acid, (3) $\mathrm{C}-\mathrm{C}$ of an aromatic ring, and (4) $\mathrm{C}=\mathrm{N}$ of imine, and (c) ${ }^{1} \mathrm{H}$ NMR spectrum of PABA modified CNCs in $d_{6}$-DMSO. 


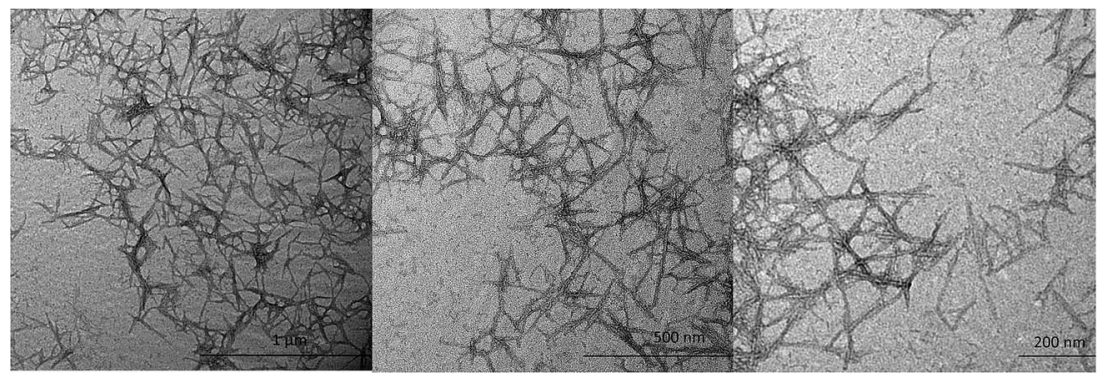

Fig. 2 TEM images of PABA-CNCs with different magnitudes.

mechanical disintegration of the PABA-modified cellulose. The width and length of PABAC nanocrystals (PABA-CNCs) were $14.07 \pm 6.09$ and $177.98 \pm 53.31 \mathrm{~nm}$, respectively. The formation of short CNCs instead of CNFs has previously been reported to occur after the disintegration of chemically modified DAC with an initial aldehyde content higher than $2 \mathrm{mmol} \mathrm{g}^{-1}$. $^{31}$ Individualization of CNCs is thought to be due to the erosion and dissolution of amorphous regions of cellulose fibers during chemical modification (during both periodate oxidation and reaction with PABA). This leads to the formation of short CNCs after mechanical disintegration. ${ }^{31}$

Based on the WAXD measurements, CrI of the PABA-CNCs was $56 \%$ (CrI of the original pulp was $57 \%{ }^{42}$ ). CrI of PABA-CNCs was similar to the results obtained previously with aminomodified CNCs (53-57\%) ${ }^{16}$ and slightly higher compared to that of bisphosphonated CNCs (42\%). ${ }^{31}$ This indicates that PABA modification has a similar effect on the crystallinity of cellulose fibers compared with reductive amination, which has been used previously to attach functional groups to CNCs.

In the literature, functional nanocelluloses are often obtained by postchemical treatment. Such treatment may involve solvent exchange and cumbersome and energy-demanding purification of nanosized cellulose entities. The nanocellulose functionalization approach proposed herein uses only water as the solvent, and the desired functional groups can be attached to cellulose prior to its disintegration. Thus, the approach has a number of advantages compared to existing nanocellulose functionalization methods.

The optical properties of the PABA-CNCs dispersed in water $(0.1-0.5 \%)$ were studied using a UV-Vis spectrometer (Fig. 3a). In the visible light range $(380-800 \mathrm{~nm})$, the transmittance of the PABA-CNC suspension (0.1\%) surpassed $85 \%$, which is similar to that of CNCs obtained by sequential periodate and chlorite oxidation $^{25}$ and significantly higher than that of alkylaminated $\mathrm{CNCs}^{16}$ obtained after reductive amination of DAC. At different concentrations of PABA-CNCs (0.1-0.5\%), the transmittance decreased, but it still exceeded $63 \%$, even at a concentration of $0.5 \%$ and a wavelength of $380 \mathrm{~nm}$. The results demonstrate the good uniformity and homogeneous nature of the suspensions of PABA-CNCs. The visual appearance of the CNC solutions at different PABA-CNC concentrations is presented in Fig. 3c.

In contrast to nanocellulose produced by periodate oxidation, a sharp decrease in the transmittance (i.e., strong absorption) of the PABA-CNCs was observed below a wavelength of around $340 \mathrm{~nm}$ at a concentration of $0.1 \%$ (Fig. 2a). A small increase in the transmittance was observed around $280 \mathrm{~nm}$ (UVC region). This phenomenon was more visible in the absorption spectra, as shown in Fig. 3b (logarithmic scale). The absorption gradually increased in the UVC region in accordance with an increase in the PABA-CNC concentration, reaching a maximum at a concentration of $0.4 \%$.

The transparency and absorption of the PABA-CNCs were compared to those of reference CNCs (Ref-CNCs), which were fabricated via chlorite oxidation of DAC. ${ }^{25}$ The transmittance of the reference CNCs was more than $80 \%$ (Fig. 2a), even at the lower end of the spectrum $(240 \mathrm{~nm})$. The results clearly show that the incorporation of PABA groups into the cellulose results in the formation of CNCs with strong UV-absorption properties. The visual appearance of the $0.5,0.4,0.3,0.2$, and $0.1 \%$ PABACNCs and $0.1 \%$ reference CNC solutions is presented in Fig. 3c.

The feasibility of PABA-CNCs as lightweight bifunctional UVactive reinforcing agents for polymeric materials was studied using poly(vinyl alcohol) (PVA). Different amounts of PABACNCs (0.5-10\% with respect to the mass of PVA) were dispersed in PVA solutions, followed by solvent casting and drying to obtain self-standing nanocomposite films. The transmittance of the films containing the different amounts of PABA-CNCs is presented in Fig. 4a. At the high end of the visible light region (600-800 nm), the addition of the PABA-CNCs to PVA had only a minor effect on the transmittance. For example, the transmittance of the film containing $0.5 \%$ of filler was identical to that of the pure PVA film. At the low end of the visible light spectrum $(380 \mathrm{~nm})$, the transmittance of the film with $10 \%$ of PABA-CNCs was around $80 \%$, indicating that a minimal amount of visible-light absorption or scattering occurred due to the presence of the PABA-CNC fillers. The visual appearance of the pure PVA film and PVA film with $5 \%$ of PABA-CNC is presented in Fig. $4 \mathrm{~b}$.

Similar to the PABA-CNC solution spectra, a sharp decrease in transmittance was observed in the UVA region (Fig. 4a). The lowest transmission of the PABA-CNC-containing films was at $280 \mathrm{~nm}$. At this wavelength, the transmittance of pure PVA was $70 \%$, whereas the transmittance of the PABA-CNCs varied from $43-0 \%$, depending on the concentration of the CNCs. The transmittance of the PABA-CNC-containing film increased in the UVC region, with the maximum transmission observed at around $240 \mathrm{~nm}$, but it was still significantly lower than that of the pure PVA film. For example, the transmission of the films 

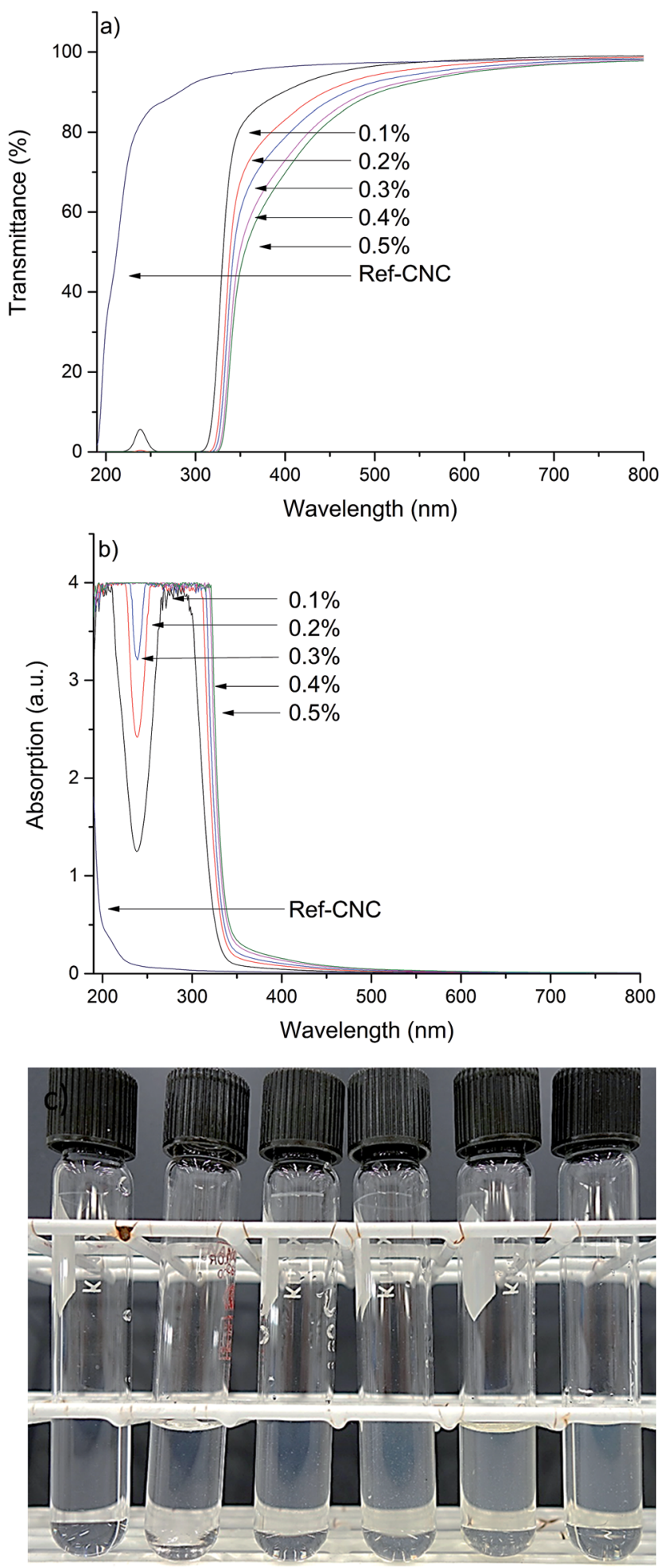

Fig. 3 (a) UV-Vis transmittance and (b) absorption spectra of the paraaminobenzoic acid-modified cellulose nanocrystal (PABA-CNC) solutions at different concentrations. The reference CNC was a $0.1 \%$ solution of CNCs obtained by the periodate oxidation-based method, without UV absorption groups (see the Experimental section). (c) The visual appearance of the $\mathrm{CNC}$ solutions: from right to left $0.5,0.4,0.3$, 0.2 , and $0.1 \%$ PABA-CNC and $0.1 \%$ Ref-CNC solutions.
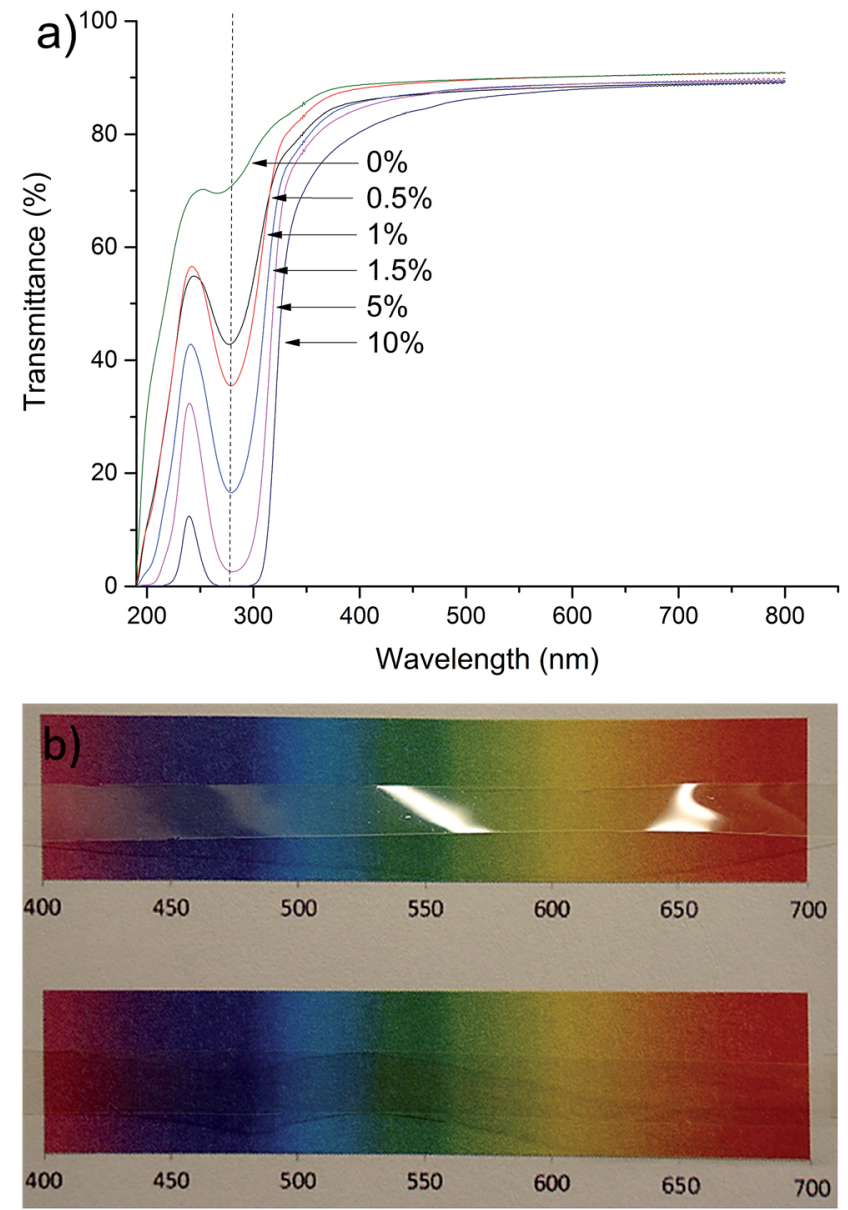

Fig. 4 (a) Transmittance of the pure PVA film and PVA films containing PABA-CNC fillers $(0.5-10 \%)$, with the dashed line indicating the minimum in the UVB region, and (b) the visual appearance of the pure PVA film (top) and PVA film with $5 \%$ of PABA-CNCs.

containing $0.5 \%$ and $10 \%$ of PABA-CNCs was $54 \%$ and $12 \%$, respectively, vs. $70 \%$ for the pure PVA film. Consequently, the PABA-CNCs functioned as efficient fillers for UV absorption when incorporated into the PVA films, particularly in the UVA and UVB regions. The latter is important because UVA wavelengths are not absorbed by the ozone layer. The increase in the transmittance in the UVC region strongly indicates that the UV absorption was due to the incorporation of the PABA groups into the cellulose and not light absorption or light scattering by aggregation of CNCs that may have formed during the film preparation. UV absorption properties of PABA-CNC reinforced PVA films were similar to those of ZnO-nanoparticle-CNF ${ }^{22}$ and lignin-CNC ${ }^{21}$ composites. However, with PABA-CNCs, slightly higher UV light selectivity over visible light was observed.

The mechanical properties of the PVA films improved when the PABA-CNCs were used as fillers. The tensile strength is shown in Fig. 5a. There was no significant difference in the tensile strength when $0.5 \%$ and $1 \%$ of PABA-CNCs were used, but the tensile strength gradually increased when the amount of PABA-CNCs was increased from $1 \%$ to $10 \%$. Compared to pristine PVA, 33\% higher tensile strength was obtained using $10 \%$ PABA-CNCs. The tensile strengths were only slightly lower 

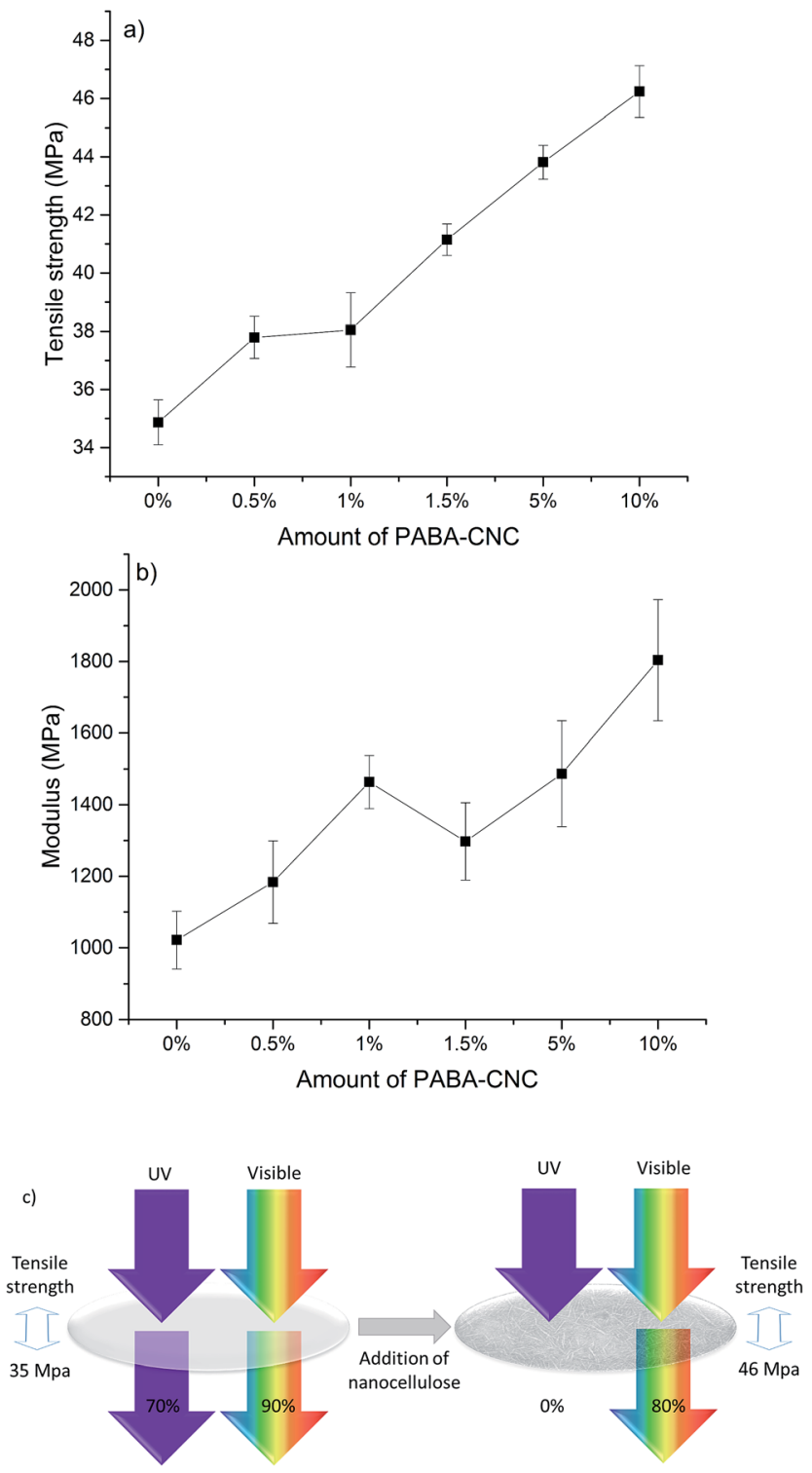

Fig. 5 (a) Tensile strength, (b) modulus of the reinforced PVA films as a function of PABA-CNCs and (c) schematic describing the UV-filtering and reinforcing action of the PABA-CNC-containing nanocomposite.

than those reported previously using reactive CNCs (RCNCs) obtained from partially carboxylated DAC containing residual aldehydes (46 MPa vs. $50 \mathrm{MPa}$ ). ${ }^{25}$ The results indicate that the residual unreacted aldehydes of PABA-CNCs functioned as cross-linking agents, resulting in improved mechanical properties. The reinforcing effect of the PABA-CNCs was similar or even higher than that reported for other nanocelluloses. ${ }^{43}$

The modulus of the PABA-CNC-reinforced PVA increased as a function of the amount of filler added (Fig. 5b), with up to a $77 \%$ higher modulus achieved using $10 \%$ of PABA-CNCs, although some variation occurred. The increase in the modulus was slightly lower than that reported previously for RCNCs, ${ }^{25}$ possibly due to the bulky PABA groups vs. the small carboxylic acid groups, but it was well in line with that found in the literature. ${ }^{43}$ The tensile testing demonstrated that in addition to the novel UV-absorption properties, PABA-CNCs provided a good reinforcing effect to the PVA-films.

Fig. 5c presents a scheme depicting the action of the reinforced composites containing the UV-absorbing PABA-CNCs. The UV-absorption properties of materials, such as those studied in the present work, are important in several areas, such as when good UV blocking and mechanical strength are required, for example, in contact lenses and sunglasses. Although the utilization of cellulose-based materials under high moisture conditions is limited, the PABA-CNCs described in the present work could be used, for example, as a filler in laminated materials. The incorporation of photoactive CNCs into naturalbased and/or biodegradable polymers could enhance the transparency and UV-absorption properties of materials, whereas a thin-layer hydrophobic laminate could improve the moisture resistance. Surface hydrophobization of photoactive CNC-containing composites with a thin layer of hydrophobic monomers could also be used to improve the moisture resistance of PABA-CNC containing materials. In addition to healthcare applications, photoactive CNCs could function in the preservation of plants as the decrease of the ozone layer and therefore the increase in passing of UV waves, especially UVB, might affect the gene stability of plants. ${ }^{44}$ Incorporating photoactive CNCs into composites and laminated materials used in greenhouses could help to protect UV-induced plant damage. The excellent mechanical properties of the photoactive CNCs developed in the present work illustrate the feasibility of PABACNCs as a filler, especially in load-bearing structures in greenhouses or in everyday wearables, such as sunglasses.

\section{Conclusion}

In this study, CNCs with excellent UV absorption properties were successfully fabricated by sequential periodate and "clicktype" imination of wood cellulose fibers. The method presented here is straightforward as the chemical modification is performed prior to the liberation of CNCs, eliminating the postpurification steps. PABA-CNCs could then be used as efficient reinforcement and UV absorbing agents for the PVA matrix. Complete elimination of UVA and UVB light was observed by addition of PABA-CNCs to PVA and even in the UVC region the transmittance of the films with added PABA-CNCs was lower than that of pure PVA. Regardless of the excellent UV absorption, the addition of PABA-CNCs exhibited only a minor effect on the transparency of the PVA films in the visible-light region. In addition, the PABA-CNCs showed a good reinforcing effect when incorporated into the PVA films. The results thus far are amongst the first ones to show simultaneous reinforcing and UV-absorbing effects of CNCs in polymeric nanocomposites. The above-mentioned features of PABA-CNCs could be exploited in numerous applications, which stipulate UV-protection qualities.

\section{Acknowledgements}

Ms Anna Tenhunen and Mr Joni Kantola are recognized for their contribution to the experimental part of the study. The 
facilities of the Center of Microscopy and Nanotechnology of the University of Oulu were utilized in this research.

\section{References}

1 H. Althues, J. Henle and S. Kaskel, Chem. Soc. Rev., 2007, 36, 1454-1465.

2 S. Li, M. M. Lin, M. S. Toprak, D. K. Kim and M. Muhammed, Nano Rev., 2010, 1, 5214-5232.

3 R. P. Rastogi, Richa, A. Kumar, M. B. Tyagi and R. P. Sinha, J. Nucleic Acids, 2010, 2010, 592980.

4 P. Gijsman, G. Meijers and G. Vitarelli, Polym. Degrad. Stab., 1999, 65, 433-441.

5 S. Madronich, R. L. McKenzie, L. O. Björn and M. M. Caldwell, J. Photochem. Photobiol., B, 1998, 46, 5-19.

6 X. An, T. W. Butler, M. Washington, S. K. Nayak and S. Kar, ACS Nano, 2011, 5, 1003-1011.

7 B. C. Heng, X. Zhao, S. Xiong, K. Woei Ng, F. Yin-Chiang Boey and J. Say-Chye Loo, Food Chem. Toxicol., 2010, 48, 17621766.

8 S. K. Hanna, R. J. Miller and H. S. Lenihan, Nanomaterials, 2014, 4, 535-547.

9 Y. Xiao, M. G. Vijver, G. Chen and W. J. G. M. Peijnenburg, Environ. Sci. Technol., 2015, 49, 4657-4664.

10 N. S. Allen, M. Edge, A. Ortega, G. Sandoval, C. M. Liauw, J. Verran, J. Stratton and R. B. McIntyre, Polym. Degrad. Stab., 2004, 85, 927-946.

11 D. Klemm, F. Kramer, S. Moritz, T. Lindström, M. Ankerfors, D. Gray and A. Dorris, Angew. Chem., Int. Ed., 2011, 50, 54385466.

12 H. P. S. Abdul Khalil, Y. Davoudpour, M. N. Islam, A. Mustapha, K. Sudesh, R. Dungani and M. Jawaid, Carbohydr. Polym., 2014, 99, 649-665.

13 Y. Habibi, L. A. Lucia and O. J. Rojas, Chem. Rev., 2010, 110, 3479-3500.

14 Z.-Y. Qin, G.-L. Tong, Y. C. Frank Chin and J.-C. Zhou, BioResources, 2011, 6, 1136-1146.

15 A. C. W. Leung, S. Hrapovic, E. Lam, Y. Liu, K. B. Male, K. A. Mahmoud and J. H. T. Luong, Small, 2011, 7, 302-305.

16 M. Visanko, H. Liimatainen, J. A. Sirviö, J. P. Heiskanen, J. Niinimäki and O. Hormi, Biomacromolecules, 2014, 15, 2769-2775.

17 H. Yang, M. N. Alam and T. G. M. van de Ven, Cellulose, 2013, 20, 1865-1875.

18 X. Qin, W. Xia, R. Sinko and S. Keten, Nano Lett., 2015, 15, 6738-6744.

19 L. Alexandrescu, K. Syverud, A. Gatti and G. ChingaCarrasco, Cellulose, 2013, 20, 1765-1775.

20 R. J. Moon, A. Martini, J. Nairn, J. Simonsen and J. Youngblood, Chem. Soc. Rev., 2011, 40, 3941-3994.
21 A. Hambardzumyan, L. Foulon, B. Chabbert and V. AguiéBéghin, Biomacromolecules, 2012, 13, 4081-4088.

22 Y. Jiang, Y. Song, M. Miao, S. Cao, X. Feng, J. Fang and L. Shi, J. Mater. Chem. C, 2015, 3, 6717-6724.

23 J. Tang, Y. Song, R. M. Berry and K. C. Tam, RSC Adv., 2014, 4, 60249-60252.

24 M. Kettunen, R. J. Silvennoinen, N. Houbenov, A. Nykänen, J. Ruokolainen, J. Sainio, V. Pore, M. Kemell, M. Ankerfors, T. Lindström, M. Ritala, R. H. A. Ras and O. Ikkala, Adv. Funct. Mater., 2011, 21, 510-517.

25 J. A. Sirviö, S. Honkaniemi, M. Visanko and H. Liimatainen, ACS Appl. Mater. Interfaces, 2015, 7, 19691-19699.

26 L. Segal, J. J. Creely, A. E. Martin and C. M. Conrad, Text. Res. J., 1959, 29, 786-794.

27 J. Sirvio, U. Hyvakko, H. Liimatainen, J. Niinimaki and O. Hormi, Carbohydr. Polym., 2011, 83, 1293-1297.

28 L. Doub and J. M. Vandenbelt, J. Am. Chem. Soc., 1947, 69, 2714-2723.

29 J. Clayden, N. Greeves and S. Warren, Organic Chemistry, OUP Oxford, 2012.

30 J. A. Sirviö, M. Visanko, O. Laitinen, A. Ämmälä and H. Liimatainen, Carbohydr. Polym., 2016, 136, 581-587.

31 J. A. Sirviö, T. Hasa, J. Ahola, H. Liimatainen, J. Niinimäki and O. Hormi, Carbohydr. Polym., 2015, 133, 524-532.

32 H. Spedding, J. Chem. Soc., 1960, 3147-3152.

33 Z. Sabzalian, M. N. Alam and T. G. M. van de Ven, Cellulose, 2014, 21, 1381-1393.

34 N. Drogat, R. Granet, V. Sol, A. Memmi, N. Saad, C. K. Koerkamp, P. Bressollier and P. Krausz, J. Nanopart. Res., 2010, 13, 1557-1562.

35 X. D. Liu, N. Nishi, S. Tokura and N. Sakairi, Carbohydr. Polym., 2001, 44, 233-238.

36 P. Calvini, G. Conio, E. Princi, S. Vicini and E. Pedemonte, Cellulose, 2006, 13, 571-579.

37 P. R. Sharma and A. J. Varma, Carbohydr. Polym., 2014, 114, 339-343.

38 L. Marin, B. Simionescu and M. Barboiu, Chem. Commun., 2012, 48, 8778-8780.

39 M. A. Ciufolini and G. O. Spencer, J. Org. Chem., 1989, 54, 4739-4741.

40 S.-H. Lee and G. I. Nikonov, Dalton Trans., 2014, 43, 88888893.

41 G. Odian, N.-I. Yang and Y. Wei, Magn. Reson. Chem., 1985, 23, 908-915.

42 J. A. Sirviö, A. Kolehmainen, H. Liimatainen, J. Niinimäki and O. E. O. Hormi, Food Chem., 2014, 151, 343-351.

43 B. Tan, Y. Ching, S. Poh, L. Abdullah and S. Gan, Polymers, 2015, 7, 2205-2222.

44 G. Ries, W. Heller, H. Puchta, H. Sandermann, H. K. Seidlitz and B. Hohn, Nature, 2000, 406, 98-101. 